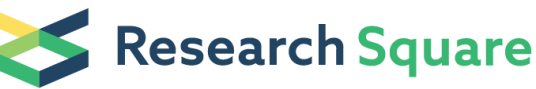 \\ Preprints are preliminary reports that have not undergone peer review. \\ They should not be considered conclusive, used to inform clinical practice, \\ or referenced by the media as validated information.
}

\section{An analysis of histopathological features of Crohn's disease in surgical specimens}

\section{Yujuan Fu}

Zhejiang University School of Medicine Sir Run Run Shaw Hospital

Xiuli Liu

University of Florida

Jiaqi Xu

Zhejiang University School of Medicine Sir Run Run Shaw Hospital

Tingting Zhong

Zhejiang University School of Medicine Sir Run Run Shaw Hospital

Junchang Jiang

Zhejiang University School of Medicine Sir Run Run Shaw Hospital

\section{Qian Cao}

Zhejiang University School of Medicine Sir Run Run Shaw Hospital

\section{Tianhong Fu}

Zhejiang University School of Medicine Sir Run Run Shaw Hospital

\section{Zhinong Jiang ( $\mathbf{0} 3200039 @ z j u . e d u . c n$ )}

Zhejiang University School of Medicine Sir Run Run Shaw Hospital https://orcid.org/0000-0003-40400407

\section{Research article}

Keywords: Crohn's disease, surgical bowel resection, histology

Posted Date: March 16th, 2021

DOI: https://doi.org/10.21203/rs.3.rs-318692/v1

License: (c) (1) This work is licensed under a Creative Commons Attribution 4.0 International License.

Read Full License 


\section{Abstract \\ Background}

The diagnosis of Crohn's disease is challenging. This study aimed to compare the histological features of Crohn's disease and non-Crohn's disease (i.e., other intestinal inflammatory diseases) in surgical specimens to identify histologic features for differential diagnosis.

\section{Methods}

We evaluated patients who were diagnosed with Crohn's disease $(n=171)$ and non-Crohn's disease $(n=$ 215) between 2010 and 2015 and underwent surgical bowel resection. The frequency of histological features in surgical resection specimens were compared between these two patient groups.

\section{Results}

Transmural inflammation, subserosal lymphoid aggregates, fissures or sinus-like structures, granulomas or granuloma-like nodules, abnormalities of the enteric nervous system, and mucosa structure alterations (i.e., muscularis mucosae thickening or mucosal atrophy with pseudopyloric gland metaplasia) were more frequent in Crohn's disease than non-Crohn's disease $(p<0.001$ for all). A set of 3 of the above pathological features had a specificity of $93.5 \%$ for Crohn's disease. Some of the above histologic features were further grouped as chronic inflammatory change that includes granulomas or granulomalike nodules, lymphoid aggregates in the muscularis propria or subserosa, fissures or sinus-like structures, and architectural abnormality (i.e., the presence of abnormal enteric nervous system and/or mucosa structure alterations). A combination of transmural inflammation, chronic inflammatory change, and architectural abnormality had a sensitivity of $92.4 \%$ and a specificity of $97.7 \%$ for Crohn's disease.

\section{Conclusions}

A combination of transmural inflammation, chronic inflammatory change, and architectural abnormality in surgical bowel resection specimens is diagnostic for Crohn's disease.

\section{Background}

Gut inflammatory bowel disease (IBD) is a group of chronic gastrointestinal disorders characterized by relapsing and remitting idiopathic inflammation of the gastrointestinal tract. The two most common types of IBDs are ulcerative colitis and Crohn's disease (CD). The diagnosis of CD is confirmed by clinical evaluation and a combination of endoscopic, histological, radiological, and/or biochemical investigations[1]. The European Crohn's and Colitis Organisation (ECCO) consensus has proposed a diagnostic criteria for $C D$ in surgical specimens. $C D$ can present as the following microscopic features in 
surgical specimens: transmural inflammation aggregated inflammatory pattern and transmural lymphoid hyperplasia; submucosal thickening (expansion by fibrosis-fibromuscular obliteration and inflammation); fissure; sarcoid granuloma (including in lymph nodes); abnormalities of the enteric nervous system (submucosal nerve fiber hyperplasia and ganglionitis); and relatively unchanged epithelia-mucin preservation [1]. Thus it has been suggested that a diagnosis of $C D$ can be made in surgically resected bowel samples when three of the above features are present in the absence of granulomas, or when an epithelioid granuloma is present with one other feature provided that specific infections are excluded $[1,2]$. However, although this set of diagnostic criteria plays an important role in $C D$ diagnosis in surgically resected bowel specimens, its sensitivity and specificity have not been investigated. The incidence of CD has been steadily increasing in China in recent years [3]. Large-scale multicenter studies examining the diagnostic sensitivity and specificity are needed to validate the above diagnostic criteria.

This study aimed to review the histologic features of CD in surgically resected bowel specimens and validate the sensitivity and specificity of a combination of the examined histologic features for $C D$ diagnosis.

\section{Methods}

\section{Study design and patients}

We evaluated 171 and 215 patients who were diagnosed with CD and non-CD, respectively, between 2010 and 2015 and who underwent surgical bowel resection. The patients were identified using the pathology database at Sir Run Run Shaw Hospital, School of Medicine, Zhejiang University. Those with tumor or acute intestinal infarction were excluded. All included patients underwent intestinal surgery and were followed up for at least 12 months.

\section{Clinical diagnosis}

CD diagnosis was confirmed by clinical, endoscopic, radiological, and histological examinations [1]. The clinical diagnosis of infectious colitis was based on positivity of the pathogenic organism, and symptom relief and endoscopy healing after 6 months of antibiotic therapy without recurrence. Other causes of enteritis such as intestinal perforation, diverticulum, chronic obstruction, fistula, mesenteric arteriovenous thrombosis, appendicitis, vascular malformation, Behçet's disease, abdominal cocoon, ectopia, mucosal prolapse, eosinophilic enteritis, and necrotizing enterocolitis were diagnosed based on clinicohistologic features.

\section{Microscopic analysis}

Surgical pathology reports and slides were retrieved and reviewed by two pathologists for the following histological features: transmural inflammation, transmural lymphoid aggregates, fissures and related structures, granulomas (including granulomas in lymph nodes) and granuloma-like nodules, 
abnormalities of the enteric nervous system, muscularis mucosae thickening, mucosal atrophy, and pseudopyloric gland metaplasia. They were defined as follows:

1. Transmural inflammation: the presence of varying degree of chronic inflammatory cells such as lymphocytes and plasma cells in all layers of the bowel wall (Figure 1A).

2. Transmural lymphoid aggregates: the presence of lymphoid aggregates, with or without germinal centers, anywhere in the bowel wall, from the mucosa to the subserosa (Figure 1A). The linear distribution of lymphoid aggregates were referred to as "the string of beads" (or "Crohn's rosary") (Figure1B) $[4,5,6]$. Transmural lymphoid aggregates were divided into two types according to the distribution: submucosa and beyond submucosa (involving muscularis propria or subserosa). Further, frequent lymphoid aggregates were defined as 3 or more per low power field [7].

3. Granulomas and granuloma-like nodules: a granuloma was defined a discrete collection of at least five epithelioid cells (activated histiocytes with homogeneous eosinophilic cytoplasm), with or without accompanying multinucleate giant cells, in the bowel wall or lymph nodes [8,9] (Figure 2A-B). Caseation should be absent. Histiocytic reaction around a ruptured crypt or isolated multinucleate giant cell were excluded. The granuloma-like nodules were composed of non-epithelioid histiocytes without multinucleated giant cells and with clear boundaries. The granuloma-like nodules in the mucosa were excluded. (Figure 2C-F).

4. Fissures and related structures: fissures were identified as deep, flask-shaped, or knife-like ulcers that extended into the submucosa or muscularis propria and were lined by plump fibroblasts, many neutrophils, histocytes and even foreign body giant cells (Figure 3A-B). Abscess was a localized collection of pus surrounded by inflamed tissue (Figure $3 \mathrm{C}$ ). Sinuses were formed by fissuring ulcers extending into or through the colonic wall and usually communicating with other fissuring ulcers extending laterally to produce a complex network. Sinuses that did not demonstrate an opening to intestinal lumen in slides, but have long or complex configuration were referred to as "sinus-like structures" (Figure 3D) [4]. Epithelium may be found in the sinus-like structures (Figure 3E) $[4,10]$.

5. Abnormalities of the enteric nervous system: this was defined as the presence of large, abnormal, irregular nerve bundles throughout the submucosa, muscularis propria or subserosa (Figure 4A-C). Perineural chronic inflammation may be seen.

6. Muscularis mucosae thickening: this was defined as increased thickness of muscularis mucosae due to hyperplasia and/or fibrosis. In some cases, the submucosa was present (Figure 5A), whereas it was obliterated in some cases (Figure 5B).

7. Mucosal atrophy: this was defined as chronic mucosal change such as villous atrophy and crypt distortion with chronic inflammatory cells (especially lymphocytes and plasma cells) in the lamina propria (Figure 5C).

8. Pseudopyloric gland metaplasia: this was defined as the presence of small clusters of small round glands in deep mucosa with clear neutral mucinous cytoplasm (Figure 5D).

\section{Statistical analysis}


The $\mathrm{c} 2$ and Fisher's exact probability tests were used to evaluate differences in the frequency of the histological parameters between the $\mathrm{CD}$ and the non-CD group. Statistical analysis comparing trends between different parameters was performed using Kendall-tau. All statistical analyses were performed using IBM SPSS Statistics Version 19.0 (IBM Corporation, Armonk, NY, USA). A $p$ value of $<0.05$ was considered statistically significant.

\section{Results}

\section{Clinical features}

Patients in the $C D$ group were significantly younger during surgical bowel resection than patients in the non-CD group (mean age $35 \pm 11.5$ years (range, $15-64$ years), vs $56 \pm 17.9$ years (range, $1-96$ years), $p<0.001)$. There was no significant difference in the male:female ratio between the $C D$ and the non-CD group (1.71 vs. $1.91, p=0.117)$. Non-CD included intestinal perforation $(n=57)$, diverticulum $(n=39)$, chronic intestinal obstruction ( $n=31)$, ulcerative colitis $(n=13)$, special infectious diseases (14), fistula $(n=12)$, mesenteric arteriovenous thrombosis $(n=7)$, appendicitis $(n=7)$, vascular malformation $(n=5)$, ectopia $(n=5)$, and other miscellaneous or unknown etiologies $(n=25)$ (Table 1$)$.

Table 1. Clinicodemographics and clinical diagnosis in $C D$ and non-CD patients 


\begin{tabular}{|c|c|c|}
\hline & $\begin{array}{l}\text { CD group } \\
(\mathrm{N}=171)\end{array}$ & $\begin{array}{l}\text { Non-CD group } \\
(\mathrm{N}=215)\end{array}$ \\
\hline Age (mean \pm SD), year & $35 \pm 11.5$ & $56 \pm 17.9$ \\
\hline Male to female ratio & 108:63 & $141: 74$ \\
\hline \multicolumn{3}{|l|}{ Type of surgical specimens } \\
\hline Small intestine resection & 63 & 101 \\
\hline lleocecal resection & 84 & 40 \\
\hline Colon resection & 15 & 73 \\
\hline Small and large intestine resection & 9 & 1 \\
\hline \multicolumn{3}{|l|}{ Type of enteropathy } \\
\hline Intestinal perforation & N/A & 57 \\
\hline Diverticulum & N/A & 39 \\
\hline Chronic intestinal obstruction & N/A & 31 \\
\hline Ulcerative colitis & N/A & 13 \\
\hline Special infectious disease & N/A & 14 \\
\hline Tuberculosis & N/A & 5 \\
\hline EB virus & N/A & 5 \\
\hline Yersinia & N/A & 4 \\
\hline Fistula & N/A & 12 \\
\hline Mesenteric arteriovenous thrombosis & N/A & 7 \\
\hline Appendicitis & N/A & 7 \\
\hline Vascular malformation & N/A & 5 \\
\hline Ectopia (stomach, pancreas) & N/A & 5 \\
\hline Behçet's disease & N/A & 1 \\
\hline Abdominal cocoon & N/A & 1 \\
\hline Mucosal prolapse & $\mathrm{N} / \mathrm{A}$ & 1 \\
\hline Eosinophilic enteritis & N/A & 1 \\
\hline Necrotizing enterocolitis & $\mathrm{N} / \mathrm{A}$ & 1 \\
\hline Uncertain etiology & N/A & 20 \\
\hline
\end{tabular}


Abbreviations: CD: Crohn's disease; non-CD: other forms of enteritis except Crohn's disease; SD: standard deviation; EB virus: Epstein-Barr virus

\section{Histological features}

The histological features in $C D$ and non-CD are shown in Table 2. The frequency of transmural inflammation was significantly higher in $\mathrm{CD}$ resection specimens than in non-CD resection specimens $(100 \%$ vs. $35.6 \%, p<0.001)$. There was no significant difference in the rate of submucosal lymphoid aggregates $(4 / 171(2.3 \%)$ vs in $8 / 215(3.7 \%), p=0.560)$, but lymphoid aggregates in the muscularis propria or subserosa were significantly more frequent in CD cases ( $89.5 \%$ vs $7.0 \%, p<0.001)$.

Table 2. Histological features of the surgically resected bowel specimens by group 


\begin{tabular}{|c|c|c|c|}
\hline Histological parameters & $\begin{array}{l}\text { CD group } \\
(\mathrm{N}=171)\end{array}$ & $\begin{array}{l}\text { Non-CD group } \\
(\mathrm{N}=215)\end{array}$ & $\begin{array}{l}p \\
\text { value }\end{array}$ \\
\hline Transmural inflammation & $171 / 171(100 \%)$ & $\begin{array}{l}70 / 215 \\
(32.6 \%)\end{array}$ & 0.000 \\
\hline \multicolumn{4}{|l|}{ Transmural lymphoid aggregates } \\
\hline Submucosa & $4 / 171(2.3 \%)$ & $8 / 215(3.7 \%)$ & $0.560^{\mathrm{a}}$ \\
\hline Muscularis propria or subserosa & $\begin{array}{l}153 / 171 \\
(89.5 \%)\end{array}$ & $15 / 215(7.0 \%)$ & 0.000 \\
\hline \multicolumn{4}{|l|}{ Granulomas and granuloma-like nodules } \\
\hline Granuloma-like nodules & 20/171 (11.7\%) & $0 / 215(0 \%)$ & $0.000^{\mathrm{a}}$ \\
\hline Granulomas & $\begin{array}{l}121 / 171 \\
(70.8 \%)\end{array}$ & $14 / 215(6.5 \%)$ & 0.000 \\
\hline Granulomas or granuloma-like nodules & $\begin{array}{l}141 / 171 \\
(82.5 \%)\end{array}$ & $14 / 215(6.5 \%)$ & 0.000 \\
\hline \multicolumn{4}{|l|}{ Fissures and related structures } \\
\hline Fissures & $94 / 171(55.0 \%)$ & $18 / 215(8.4 \%)$ & 0.000 \\
\hline Abscesses & $31 / 171(18.1 \%)$ & $\begin{array}{l}47 / 215 \\
(21.7 \%)\end{array}$ & 0.364 \\
\hline Sinus-like structures & $80 / 171(46.8 \%)$ & $4 / 215(1.7 \%)$ & $0.000^{\mathrm{a}}$ \\
\hline Fissures or sinus-like structures & $\begin{array}{l}125 / 171 \\
(73.1 \%)\end{array}$ & $\begin{array}{l}22 / 215 \\
(10.2 \%)\end{array}$ & 0.000 \\
\hline Abnormalities of the enteric nervous system & $81 / 171(47.4 \%)$ & $7 / 215(3.3 \%)$ & 0.000 \\
\hline \multicolumn{4}{|l|}{ Muscularis mucosae thickening } \\
\hline Submucosa presence & 28/171 (16.4\%) & $1 / 215(0.5 \%)$ & $0.000^{\mathrm{a}}$ \\
\hline Submucosa obliteration & $\begin{array}{l}110 / 171 \\
(64.3 \%)\end{array}$ & $1 / 215(0.5 \%)$ & $0.000^{a}$ \\
\hline Submucosa presence or obliteration & $\begin{array}{l}138 / 171 \\
(80.7 \%)\end{array}$ & $2 / 215(0.9 \%)$ & $0.000^{\mathrm{a}}$ \\
\hline \multicolumn{4}{|l|}{ Mucosa structural alterations } \\
\hline Mucosal atrophy & $\begin{array}{l}170 / 171 \\
(99.4 \%)\end{array}$ & $\begin{array}{l}49 / 215 \\
(22.8 \%)\end{array}$ & 0.000 \\
\hline Pseudopyloric gland metaplasia & $\begin{array}{l}150 / 171 \\
(87.7 \%)\end{array}$ & $20 / 215(9.3 \%)$ & 0.000 \\
\hline Mucosal atrophy with pseudopyloric gland & 149/171 & $16 / 215(7.4 \%)$ & 0.000 \\
\hline
\end{tabular}


a. Fisher's exact probability tests

Abbreviations: CD: Crohn's disease; non-CD: other forms of enteritis except Crohn's disease

Granulomas were significantly more present in CD cases than in non-CD cases $(121 / 171$ (70.8\%) vs. $6.5 \%, p<0.001)$. There were $6 / 171$ (3.5\%) CD patients who had granulomas only in the lymph nodes. Granuloma-like nodules were present in 20/171 (11.70\%) of CD cases but none of the 215 non-CD cases $(p<0.001)$.

Fissures $(55.0 \%$ vs. $8.4 \%, p<0.001)$ and sinus-like structures $(46.8 \%$ vs. $1.9 \%, p<0.001)$ were more frequent in $C D$ cases. Meanwhile the frequency of abscesses was not significantly different (18.1\% vs. $21.9 \%$, $p=0.364)$.

Muscularis mucosae thickening with intact submucosa $(16.4 \%$ vs. $0.5 \%, p<0.001)$ or obliterated submucosa $(64.3 \%$ vs. $0.5 \%, p<0.001)$ was also more common in $C D$ than in non-CD cases.

Abnormalities of the enteric nervous system were more common in CD than in non-CD cases $(47.4 \%$ vs. $3.3 \%, p<0.001)$. Mucosal atrophy $(99.4 \%$ vs. $22.8 \%, p<0.001)$ and pseudopyloric gland metaplasia $(87.7 \%$ vs. $9.3 \%, p<0.001$, respectively) were also significantly more common in CD.

Correlation analysis revealed a significant correlation between muscularis mucosae thickening and mucosal atrophy (Kendall's tau_b correlation coefficient $0.753, p<0.001$ ). Therefore, we combined them into one pathological index, that is, mucosa structure alterations (muscularis mucosae thickening or mucosal atrophy with pseudopyloric gland metaplasia), and this feature was more common in CD patients than in non-CD patients $(93.0 \%[159 / 171]$ vs. $7.9 \%[17 / 215], p<0.001)$.

Further analysis revealed that the presence of at least 3 of the 6 studied features (transmural inflammation, lymphoid aggregates in the muscularis propria or subserosa, granulomas or granulomalike nodules, fissures or sinus-like structures, abnormalities of the enteric nervous system, and mucosa structure alterations (muscularis mucosae thickening or mucosal atrophy with pseudopyloric gland metaplasia)) had a sensitivity of $98.8 \%$ and a specificity of $93.5 \%$ for distinguishing CD from non-CD cases (Table 3).

Table 3. Diagnostic value of two composite histological features in distinguishing CD from non-CD cases 


\begin{tabular}{|c|c|c|c|c|c|c|}
\hline No. of histological features & CD & $\begin{array}{l}\text { Non- } \\
\text { CD }\end{array}$ & $\begin{array}{l}\mathrm{Se} \\
\text { (\%) }\end{array}$ & $\begin{array}{l}\text { Sp } \\
(\%)\end{array}$ & PPV\% & NPV\% \\
\hline${ }^{3} 3$ of 6 histological features ${ }^{a}$ & $169 / 171$ & $14 / 215$ & 98.8 & 93.5 & 92.4 & 99.0 \\
\hline $\begin{array}{l}\text { Transmural inflammation }+{ }^{3} 1 \text { feature of } \\
\text { chronic inflammation changes }{ }^{b}+{ }^{3} 1 \text { feature } \\
\text { of architectural abnormalities }{ }^{c}\end{array}$ & $158 / 171$ & $5 / 215$ & 92.4 & 97.7 & 96.9 & 94.2 \\
\hline \multirow{3}{*}{\multicolumn{7}{|c|}{$\begin{array}{l}\text { a. The } 6 \text { histological features were as follows: transmural inflammation; granulomas or granuloma- } \\
\text { like nodules; lymphoid aggregates in the muscularis propria or subserosa; fissures or sinus-like } \\
\text { structures; abnormalities of the enteric nervous system; mucosa structure alterations (muscularis } \\
\text { mucosae thickening or mucosal atrophy with pseudopyloric gland metaplasia) } \\
\text { b. The chronic inflammatory changes were as follows: granulomas or granuloma-like nodules; } \\
\text { lymphoid aggregates in the muscularis propria or subserosa; fissures or sinus-like structures } \\
\text { c: The architectural abnormalities were as follows: abnormalities of the enteric nervous system; } \\
\text { mucosa structure alterations (muscularis mucosae thickening or mucosal atrophy with pseudopyloric } \\
\text { gland metaplasia) }\end{array}$}} \\
\hline & & & & & & \\
\hline & & & & & & \\
\hline \multicolumn{7}{|c|}{$\begin{array}{l}\text { Abbreviations: CD: Crohn's disease; non-CD: other forms of enteritis except Crohn's disease; No.: } \\
\text { number; Se: sensitivity; Sp: specificity; PPV: positive predictive value; NPV: negative predictive value }\end{array}$} \\
\hline
\end{tabular}

Then, we simplified the 5 studied histological features other than transmural inflammation into two categories: (1) chronic inflammatory changes and (2) architectural abnormalities. Chronic inflammatory changes were characterized by the presence of granulomas/granuloma-like nodules, lymphoid aggregates in the muscularis propria or subserosa, and/or fissures/sinus-like structures. Meanwhile, architectural abnormalities were characterized by the presence of abnormalities of the enteric nervous system and/or mucosa structure alterations (muscularis mucosae thickening or mucosal atrophy with pseudopyloric gland metaplasia). A combination of transmural inflammation, chronic inflammatory changes, and architectural abnormalities had a sensitivity of $92.4 \%$ and specificity of $97.7 \%$ for distinguishing $C D$ from non-CD cases.

\section{Discussion}

The diagnosis of $C D$ is based on a combination of clinical, endoscopic, radiological, and histological parameters. Although the ECCO consensus had proposed diagnostic recommendations for Crohn's surgical specimens, no study has evaluated its diagnostic value [1]. Although previous studies have reported the histological features in Crohn's surgical specimens [11, 12], thus far, no study has compared the histological features between $C D$ and non-CD. In the present study, we investigated the combination of histological features that can be useful for differential diagnosis of $C D$ from other forms of enteritis.

Univariate analysis showed that transmural inflammation, lymphoid aggregates in the muscularis propria or subserosa, granulomas or granuloma-like nodules, fissures or sinus-like structures, abnormalities of 
the enteric nervous system, mucosa structure alterations (e.g., muscularis mucosae thickening or mucosal atrophy with pseudopyloric gland metaplasia) are diagnostic for $C D$, consistent with previous studies $[11,12]$. Other features such as abscesses and submucosal lymphoid aggregates were of little value in distinguishing $C D$ and non-CD. Granulomas are characteristic findings in $C D$ and have been reported to be present in $52-75 \%$ of resection specimens $[11,13,14,15]$. In line with the literature, granulomas were found in $70.8 \%$ of $C D$ cases (including 6 cases whose granulomas were only in the lymph nodes) in this study.

We also compared the frequency and characteristics of granuloma-like nodules between $C D$ and non-CD cases and found that they were only present in $C D$, although the overall frequency was low $(11.7 \%)$. These results indicate that the presence of granuloma-like nodules in the submucosa and beyond in surgically resected specimens could indicate $C D$ and thus the specimen should be thoroughly investigated for further evidence.

Hypertrophy of nerve fibers accompanied by infiltration of chronic inflammatory cells is the most primary abnormality of the enteric nervous system. Although nerve hypertrophy is found in less than $50 \%$ of $C D$ cases, it has high specificity as it only occurs in $3.3 \%$ of non-CD cases. Thickening of the muscularis mucosae by hyperplasia or fibrosis with or without submucosal obliteration is also common in surgical specimens of $\mathrm{CD}$. The results showed that regardless of whether the submucosa was obliterated or not, muscularis mucosae thickening in a surgically resected bowel specimen should prompt careful search for more evidence of $\mathrm{CD}$.

We further examined two combinations of the six histologic features examined in this study, namely, (1) transmural inflammation; (2) lymphoid aggregates in the muscularis propria or subserosa; (3) granulomas or granuloma-like nodules; (4) fissures or sinus-like structures; (5) abnormalities of the enteric nervous system; and (6) mucosa structure alterations (muscularis mucosae thickening or mucosal atrophy with pseudopyloric gland metaplasia). The results showed that the presence of at least 3 of the above 6 features in the surgically resected bowel specimens had a sensitivity of $98.8 \%$ and specificity of 93.5\% for distinguishing CD from non-CD cases.

Further, we evaluated the diagnostic value of the combination of transmural inflammation, one chronic inflammatory change (i.e., granulomas/granuloma-like nodules, lymphoid aggregates in the muscularis propria or subserosa, or fissures or sinus-like structures), and one structural abnormality (i.e., abnormalities of the enteric nervous system or mucosa structure alterations that include muscularis mucosae thickening or mucosal atrophy with pseudopyloric gland metaplasia). This simplified combination had a sensitivity of $92.4 \%$ and a specificity of $97.7 \%$ in diagnosing CD. The 5 non-CD cases misclassified as CD using this combination included 2 cases of tuberculosis, 1 case of ulcerative colitis, 1 case of chronic obstruction, and 1 case of uncertain etiology. Chronic active Epstein-Barr virus infective enteritis may present with CD-like features such as transmural inflammation, fissuring ulcers, and lymphoid aggregates in intestinal wall[16]. However, all five cases of chronic active Epstein-Barr virus 
infective enteritis in this study were correctly classified as non-CD cases using this simplified combination.

The strengths of our study include the relatively large sample size from a single tertiary medical center with expertise in IBD care. This allowed for a relatively uniform tissue sampling of the surgically resected specimens. Further, all patients were followed up for at least 12 months. In addition, all slides were independently reviewed in detail by two pathologists experienced in IBD pathology. However, our study also had some limitations that need to be considered when interpreting the findings. First, data availability and quality were limited to those available within the medical records. For example, some cases lacked detailed information on medical treatment prior to surgical bowel resection. In addition, macroscopic features were not thoroughly documented in the surgical pathology report and thus could not be included in this study. Further, all patients were from a tertiary medical center, and this may have introduced referral bias. Accordingly, conclusions from this study may not be applicable to other practice settings. Finally, slides were only reviewed by two pathologists. Despite these limitations, we believe that this study is valuable because it provides important information for CD diagnosis in surgically resected bowel specimens. Large-scale prospective studies are needed to confirm our findings and to standardize the diagnosis of $C D$ in surgically resected bowel specimens, which would be important and particularly relevant in developing countries where the incidence of $C D$ is increasing but the pathological expertise needs to be improved.

\section{Conclusion}

Our study highlights the histological features prevalent in surgically resected CD bowel diseases. In addition to clinical, endoscopic, and radiographic information, a combination of histologic features including transmural inflammation, chronic inflammatory changes, and architectural alterations in surgically resected bowel specimens helps distinguish CD from non-CD.

\section{Abbreviations}

IBD: Inflammatory bowel disease; CD: crohn's disease; ECCO: European Crohn's and Colitis Organisation; non-CD: other forms of enteritis except Crohn's disease; SD: standard deviation; EB virus: Epstein-Barr virus; No.: number; Se: sensitivity; Sp: specificity; PPV: positive predictive value; NPV: negative predictive value.

\section{Declarations}

\section{Acknowledgements}

Not applicable.

\section{Authors' contributions}


ZJ and TF made substantial contributions to the conception and design of this study; YF wrote the manuscript; JX, TZ, JJ kindly provided some advices; QC provided the clinical advices; ZJ and XL revised the manuscript for important intellectual content; ZJ gave final approval to submit the manuscript for publication.

\section{Funding}

This work was supported by the National Natural Science Foundation of China [grant number 81800459].

\section{Availability of data and materials}

All data generated or analyzed during this study are included in the article.

\section{Ethics approval and consent to participate}

The retrospective study was approved by the ethics committee of our hospital (NO. 20180226-51). Individual consent was not necessary according to local ethical regulation in a retrospective investigation based on the hospital data management system as performed here.

\section{Consent for publication}

Not applicable.

\section{Competing interests}

The authors declare that they have no competing interests.

\section{Author details}

${ }^{1}$ Department of pathology, Sir Run Run Shaw Hospital, School of Medicine, Zhejiang University, Hangzhou, China. ${ }^{2}$ Department of Pathology, Immunology, and Laboratory Medicine, University of Florida, Gainesville, FL, USA. ${ }^{3}$ Department of Gastroenterology, Sir Run Run Shaw Hospital, School of Medicine, Zhejiang University, Hangzhou, China.

\section{References}

[1] Van Assche G, Dignass A, Panes J, et al. The second European evidence-based Consensus on the diagnosis and management of Crohn's disease: Definitions and diagnosis. J Crohns Colitis. 2010;4:7-27.

[2] Magro F, Langner C, Driessen A, et al. European consensus on the histopathology of inflammatory bowel disease. J Crohns Colitis. 2013;7:827-51.

[3] Li Y, Chen B, Gao X, et al. Current diagnosis and management of Crohn's disease in China: results from a multicenter prospective disease registry. BMC Gastroenterol. 2019;19:145. 
[4] Robert Riddell DJ. Lewin, Weinstein, and Riddell's gastrointestinal pathology and its clinical implications. 2012.

[5] Greenson JK. Diagnostic Pathology : Gastrointestinal. 2009.

[6] Odze RDGJ. Surgical Pathology of the GI Tract, Liver, Biliary Tract and Pancreas. 2008.

[7] Schaeffer DF, Walsh JC, Kirsch R, et al. Distinctive histopathologic phenotype in resection specimens from patients with Crohn's disease receiving anti-TNF-alpha therapy. Hum Pathol. 2014;45:1928-35.

[8] Jenkins D, Balsitis M, Gallivan S, et al. Guidelines for the initial biopsy diagnosis of suspected chronic idiopathic inflammatory bowel disease. The British Society of Gastroenterology Initiative. J Clin Pathol. 1997;50:93-105.

[9] Seldenrijk CA, Morson BC, Meuwissen SG, et al. Histopathological evaluation of colonic mucosal biopsy specimens in chronic inflammatory bowel disease: diagnostic implications. Gut. 1991;32:1514-20.

[10] Fenoglio-Preiser CM. Gastrointestinal pathology: an atlas and text. Wolters Kluwer/Lippincott Williams \& Wilkins, 2008.

[11] Soucy G, Wang HH, Farraye FA, et al. Clinical and pathological analysis of colonic Crohn's disease, including a subgroup with ulcerative colitis-like features. Mod Pathol. 2012;25:295-307.

[12] Cayci M, Bostanci EB, Turhan N, et al. The analysis of clinico-pathologic characteristics in patients who underwent surgery due to stricturing and non-perineal fistulizing forms of Crohn's disease: a retrospective cohort study. Int J Surg. 2015;15:49-54.

[13] Pierik M, De Hertogh G, Vermeire S, et al. Epithelioid granulomas, pattern recognition receptors, and phenotypes of Crohn's disease. Gut. 2005;54:223-7.

[14] Brinar M, Vermeire S, Cleynen I, et al. Genetic variants in autophagy-related genes and granuloma formation in a cohort of surgically treated Crohn's disease patients. J Crohns Colitis. 2012;6:43-50.

[15] Heresbach D, Alexandre JL, Branger B, et al. Frequency and significance of granulomas in a cohort of incident cases of Crohn's disease. Gut. 2005;54:215-22.

[16] Liu R, Wang M, Zhang L, et al. The clinicopathologic features of chronic active Epstein-Barr virus infective enteritis. Mod Pathol. 2019;32:387-395.

\section{Figures}



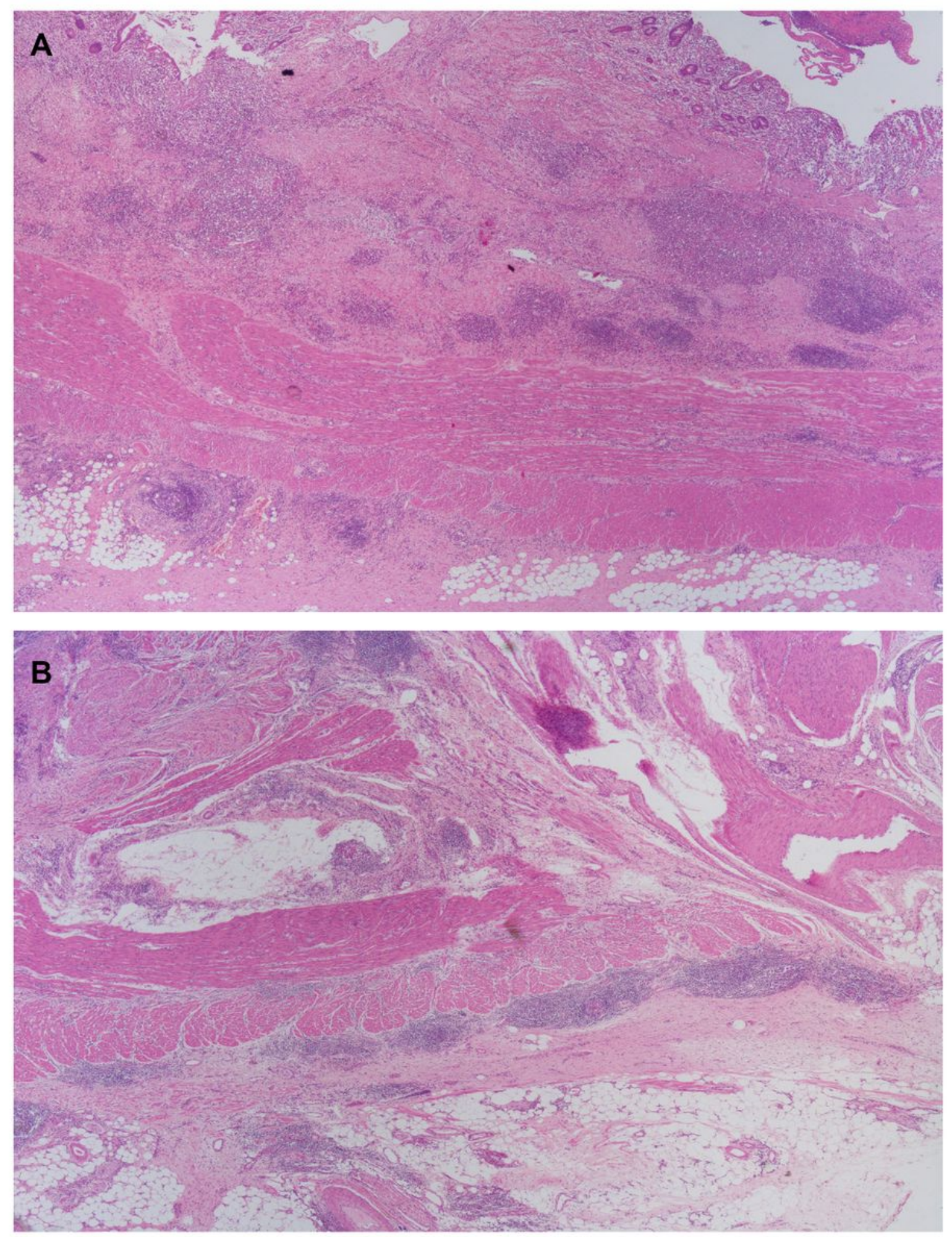

\section{Figure 1}

Transmural inflammation and transmural lymphoid aggregates. (A) Transmural inflammation and lymphoid aggregates HE×20. (B) "The string of beads" in the subserosa HE×20. 

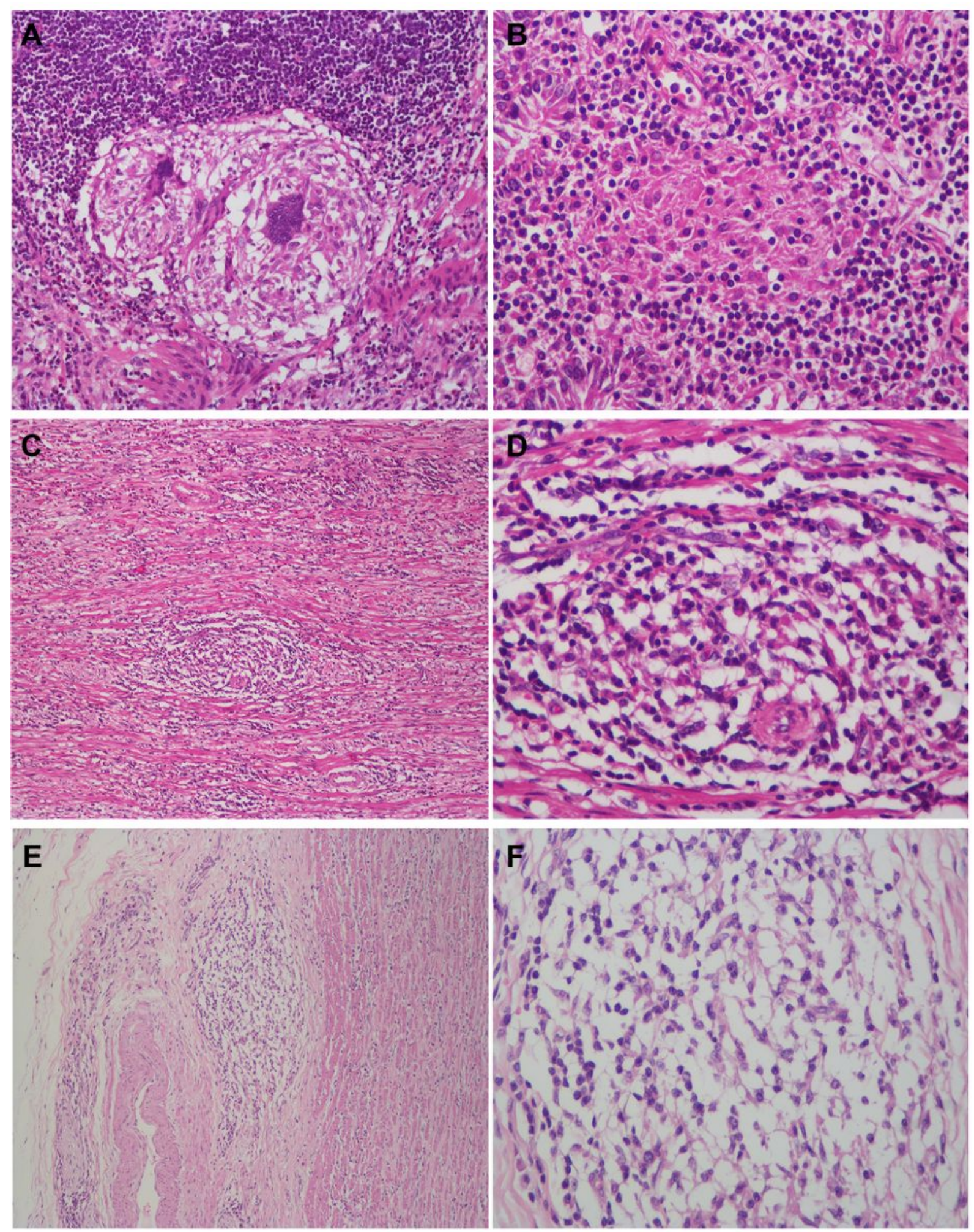

\section{Figure 2}

Granulomas and granuloma-like nodules. Granuloma is a discrete collection of epithelioid cells (activated histiocytes with homogeneous eosinophilic cytoplasm), with accompanying multinucleate giant cells $\mathrm{HE} \times 200(\mathrm{~A})$, or without accompanying multinucleate giant cells HEx40 (B). Granuloma-like nodule is a well-defined nodule of non-epithelioid histiocytes in the muscularis propria (C HEx100, D HEx400) and subserosa (E HEx100, F HEx400). 

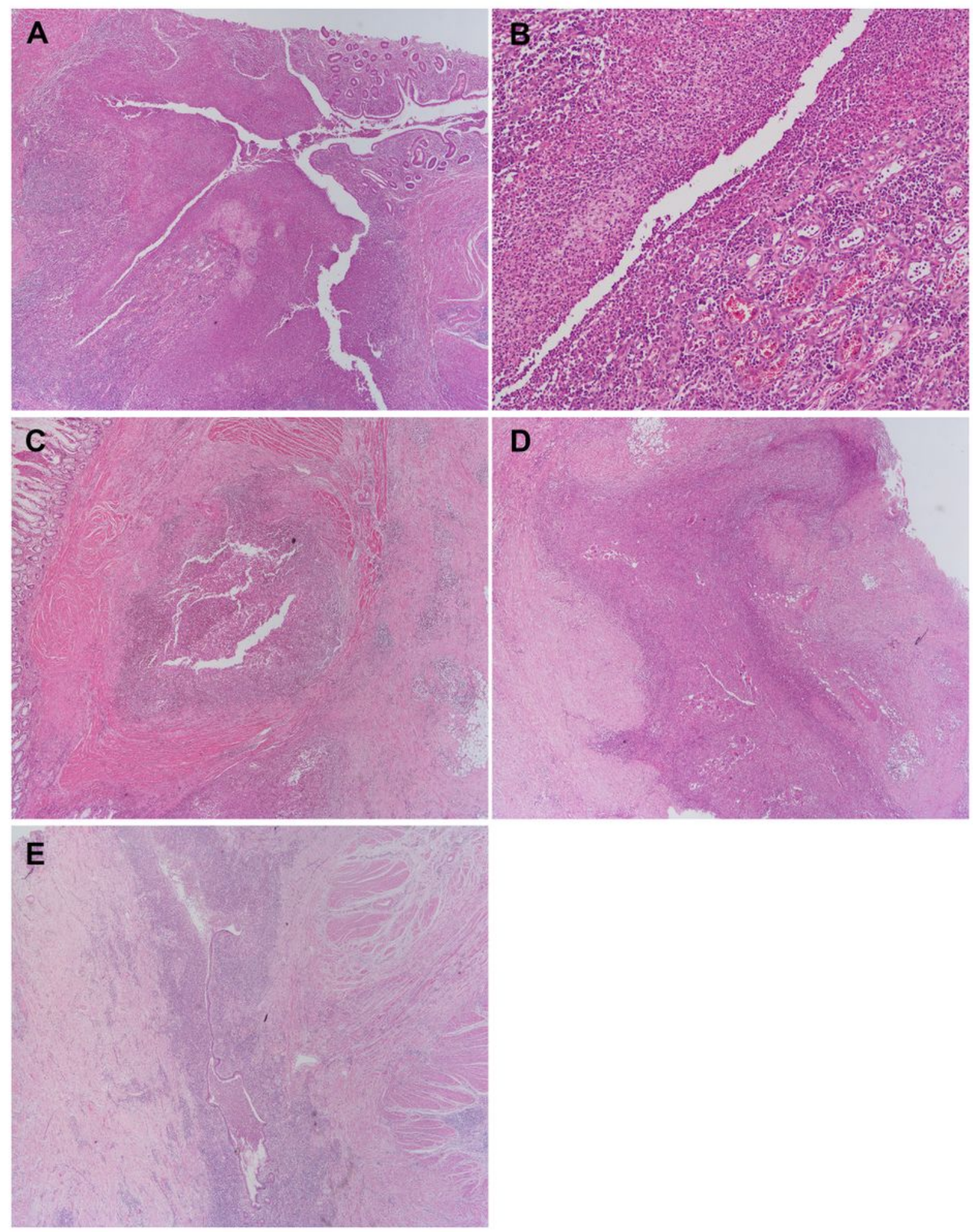

\section{Figure 3}

Fissures and related structures. Fissures are deep, flask-shaped, or knife-like ulcers that extend into the submucosa HEx20 (A) and is lined by numerous neutrophils HEx100 (B). Abscess is a localized collection of pus surrounded by inflamed tissue HEx20 (C). Some sinuses do not demonstrate an opening to intestinal lumen on slides, but those with long or complex configurations are considered a sinus-like structure HE×20 (D). Sometimes, epithelium lines the sinus-like structure HE×20 (E). 

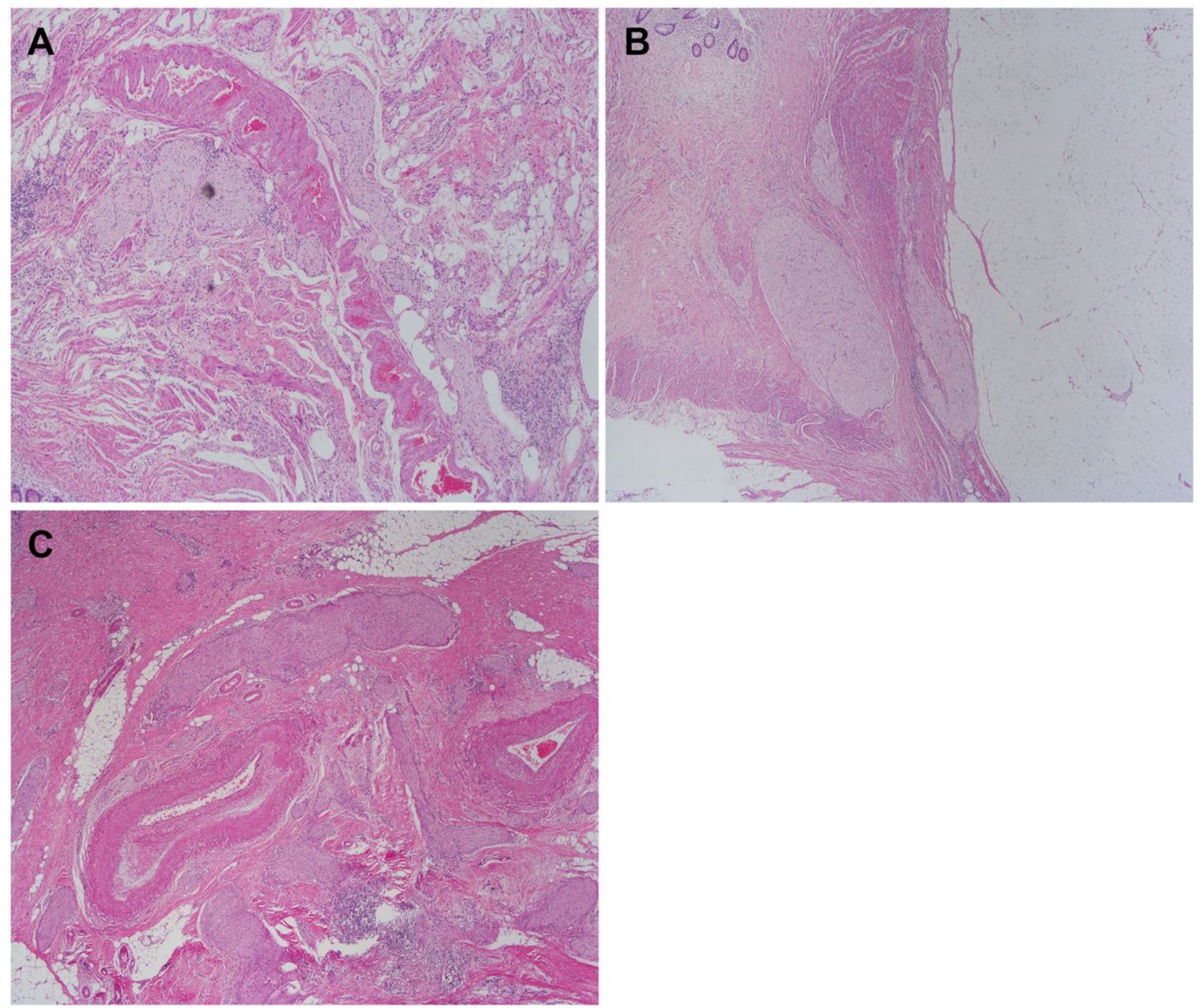

\section{Figure 4}

Abnormalities of the enteric nervous system. Large, abnormal, and irregular nerve bundles are present in the submucosa HE×40 (A), muscularis propria HE×20 (B), or subserosa HE×20 (C). 


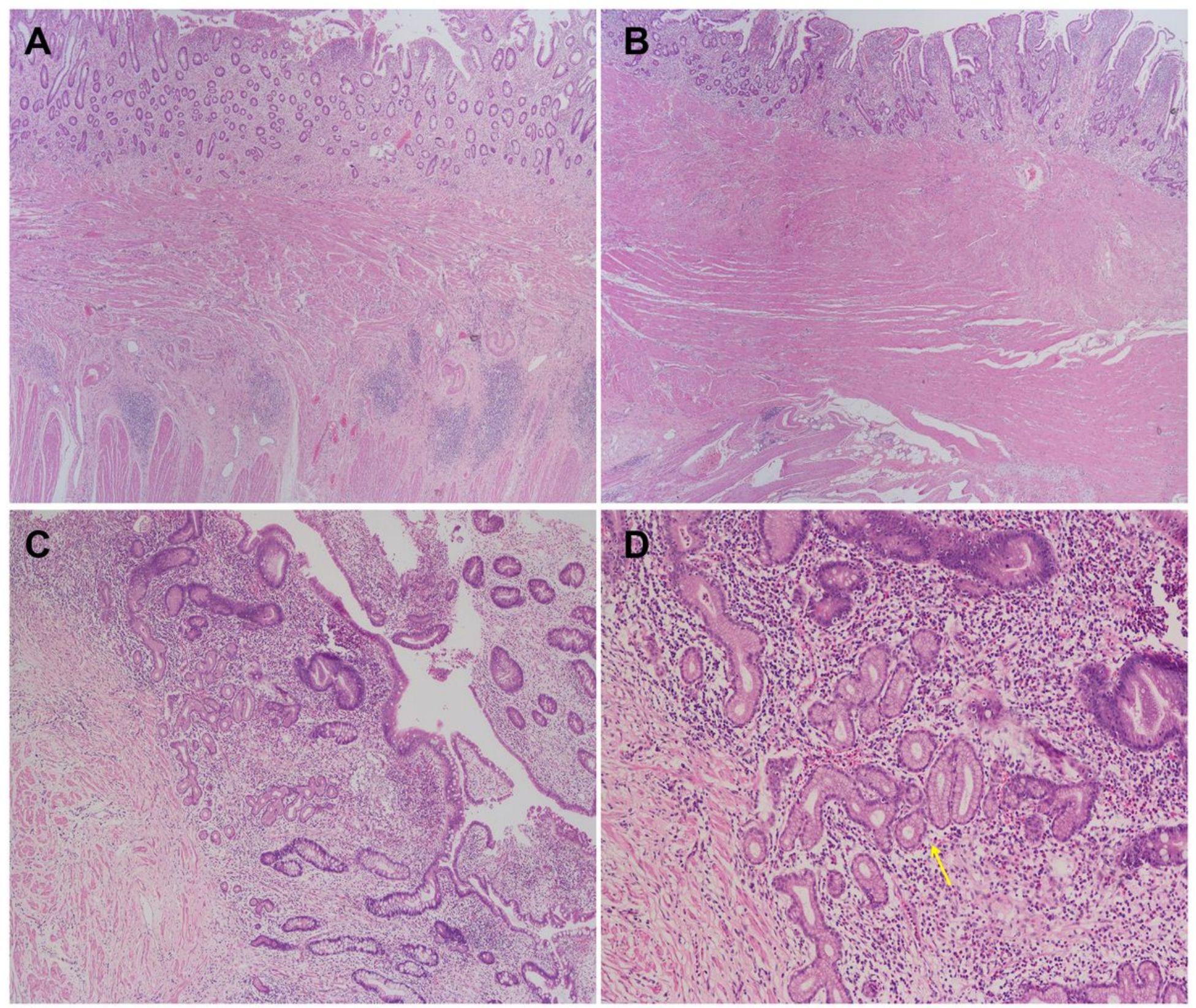

\section{Figure 5}

Architectural abnormalities. Muscularis mucosae is thickened by hyperplasia or fibrosis. Sometime, the submucosa is still present HE×20 (A), or obliterated HE×20 (B). Mucosal atrophy with pseudopyloric gland metaplasia (arrow) (C HEx40, D HEx100). 